\title{
Diffusion in simple fluids
}

\author{
by R. J. SPEEDY \\ Chemistry Department, Victoria University, Wellington, \\ New Zealand \\ F. X. PRIELMEIER, T. VARDAG, E. W. LANG \\ and H.-D. LÜDEMANN \\ Institut für Biophysik und Physikalische Biochemie, \\ Universität Regensburg, D8400 Regensburg, West Germany
}

(Received 8 August 1988; accepted 29 September 1988)

\begin{abstract}
Computed self diffusion coefficients for the Lennard-Jones and hard sphere fluids are related by

$$
D_{\mathrm{LJ}}=D_{\mathrm{HS}}\left(\sigma_{\mathrm{B}}\right) \exp \left(-\varepsilon / 2 k_{\mathrm{B}} T\right)
$$

where $\sigma_{\mathrm{B}}=\sigma_{\mathrm{L}}\left(2 /\left[1+\sqrt{ }\left(1+2 k_{\mathrm{B}} T / \varepsilon\right)\right]\right)^{1 / 6}$, the effective hard sphere diameter, is the (average) distance of closest approach in collisions between molecules which interact with the positive part of the $L J$ potential, and the Arrhenius term reflects the influence of the negative part. $\sigma_{\mathrm{LS}}$ and $\varepsilon$ are the size and well depth parameters. Measured diffusion coeflicients of the halomethane liquids are reproduced by the equation over wide ranges of temperature and density and do not reveal any influence of the inelastic effects associated with molecular anisotropy.
\end{abstract}

\section{Introduction}

Boltzmann [1] stated that 'Between the centres of two molecules there acts an attractive force (the van der Waals cohesion force) which vanishes at macroscopic distances and varies so slowly with increasing distance that it may be considered constant within distances large compared to the average separation of two neighbouring molecules. Consequently the van der Waals cohesion forces exerted on each molecule in the interior of the container are very nearly equal in all directions in space, and they balance each other in such a way that the motion of the individual molecule is like that of the usual gas molecule, and is not noticeably modified by the cohesion forces.'

That approximation underlies the modern day perturbation theories of liquids $[2,3,4]$ which successfully relate the thermodynamic properties of a real fluid to those of a corresponding purely repulsive system (which Boltzmann refers to above as 'the usual gas'), using the assumption that attractive forces do not affect the structure. It also underlies those interpretations of the dynamic properties of fluids which assume that diffusion, for example, is not influenced by attractive forces $[5,6$, 7].

Boltzmann also discussed the collisional trajectories of molecules which interact with a purely repulsive force law. He noted that if the molecules are replaced by elastic (hard) spheres, whose diameter is chosen as the distance of closest approach of the colliding pair, then the trajectories 'are indeed quantitatively different but not essentially qualitatively different' [8]. 
The two statements quoted indicate that Boltzmann foresaw that the structure and dynamics of simple real fluids could be modelled by those of a corresponding hard sphere system. Furthermore, he provides a prescription for estimating the diameter of the hard spheres.

One implication of those ideas is that the self diffusion coefficient of a simple real fluid might be represented by

$$
D=D_{\mathrm{Hs}},
$$

where $D_{\mathrm{HS}}\left(T, \rho, \sigma_{\mathrm{B}}\right)$ is the self diffusion coefficient of a fluid of hard spheres at the same number density $\rho=N / V$, temperature $T$, and where the diameter of the hard spheres $\sigma_{\mathbf{B}}$ is chosen to be (some form of average over collisions of) the distance of closest approach of colliding pairs.

Equation (1) is familiar but Boltzmann's prescience is often overlooked and the assumptions which underlie it are seldom so clearly stated. His prescription for determining $\sigma_{\mathrm{B}}$ has not, to our knowledge, been tested.

The motivation for our work is to interpret the magnitude and the temperature and density dependence of diffusion in real fluids. We first show that for some selected systems, methane [9] and a purely repulsive model system defined by the Weeks, Chandler, Andersen core potential [10], Boltzmann's ideas lead directly to quantitatively accurate predictions. We then analyse computer simulation data for the square-well [9] and Lennard-Jones $[10,12]$ model and conclude that the cohesive part of the inter molecular potential plays a significant role in reducing diffusion. When that effect is accounted for it becomes possible to represent the experimentally measured diffusion coefficients of the halomethanes, the simplest class of fluids for which precise and extensive data are available [13-17], using two fitted parameters, the Lennard-Jones size and well depth, to characterise the molecules.

Our work differs in several ways from previous studies which have had similar objectives $[5,6,18]$ : we use an improved expression for the hard sphere diffusion coefficient [19]; we follow Boltzmann's prescription for choosing the hard sphere diameter (and show that the Weeks, Chandler, Andersen prescription [4], which works for the structure and thermodynamic properties, overestimates the diameter appropriate for interpreting diffusion); and we show that when proper allowance is made for the influence of the cohesive energy it is not necessary (for the halomethanes) to attribute any reduction in diffusion to inelastic effects such as proposed by Chandler [7] in his 'rough hard sphere' model.

\section{The hard sphere diffusion coefficient $D_{\mathrm{HS}}$}

Values of $D_{\mathrm{HS}}$ from molecular dynamics simulations are re-assessed in [19] and represented by

$$
D_{\mathrm{HS}}=\left(D_{0} / n\right)(1-n / 1.09)\left(1+n^{2}\left(0.4-0.83 n^{2}\right)\right) \text {, }
$$

where $n=\rho \sigma^{3}$ and $D_{0}=(3 / 8) \sigma\left(k_{\mathrm{B}} T / \pi m\right)^{1 / 2} . \sigma$ is the sphere diameter, $k_{\mathrm{B}} T$ is the Boltzmann's constant times the temperature and $m$ is the mass of a sphere.

Equation (2) is accurate from the ideal gas limit, where it is exact, to the highest density, $\rho \sigma^{3}=1.08$, at which the fluid has been studied. The density of the hard sphere fluid at its freezing point is $\rho \sigma^{3}=0.94$ and there are indications that the fluid 
state ends in a glass transition $[19,20]$ at $\rho \sigma^{3}=1 \cdot 09$, where $D_{\mathrm{HS}}$ extrapolates to zero according to equation (2).

\section{Boltzmann's hard sphere diameter $\sigma_{B}$}

The energy of collision between an isolated pair of molecules, $E_{\mathrm{c}}$, is the kinetic energy of the pair along the line of their centres, in their centre-of-mass-fixed frame of reference, long before or long after impact. At the distance of closest approach the molecules are stationary (in the above reference frame) and the kinetic energy $E_{\mathrm{c}}$ appears as the potential energy of their interaction. If $u(r)$ is the potential energy of interaction of the molecules when they are separated by $r$, then the distance of closest approach, which we call Boltzmann's diameter $\sigma_{\mathrm{B}}$, is determined by the condition

$$
u\left(\sigma_{\mathrm{B}}\right)=E_{\mathrm{c}} .
$$

Chapman and Cowling [21] show that, for a hard sphere system, the value of $E_{c}$, averaged over all collisions, is

$$
E_{\mathrm{c}}=2 k_{\mathrm{B}} T \text {. }
$$

On that basis we identify $\sigma_{\mathrm{B}}$ as the (smallest) value which satisfies

$$
u\left(\sigma_{\mathrm{B}}\right)=2 k_{\mathrm{B}} T \text {. }
$$

Equation (4) is exact for hard spheres at all densities and (3) is exact for a collision between a pair of colliding molecules whose relative velocity is uncorrelated with the positions of neighbouring molecules, but, clearly, some assumptions are needed to justify their application to real liquids. Rather than attempt a fundamental derivation we examine the validity of equation (5) empirically, by using it to predict the diffusion coefficient, $D_{\text {wCA }}$, of a model fluid described by the purely repulsive Weeks, Chandler, Andersen core potential,

$$
u(r)= \begin{cases}4 \varepsilon\left(\left(\sigma_{\mathrm{LJ}} / r\right)^{12}-\left(\sigma_{\mathrm{LJ}} / r\right)^{6}\right)+\varepsilon, & r \leqslant 2^{1 / 6} \sigma_{\mathrm{LJ}}, \\ 0, & r>2^{1 / 6} \sigma_{\mathrm{LJ}},\end{cases}
$$

for which equation (5) gives

$$
\sigma_{\mathrm{B}}=\sigma_{\mathrm{LJ}}\left(2 /\left[1+\sqrt{ }\left(2 k_{\mathrm{B}} T / \varepsilon\right)\right]\right)^{1 / 6} .
$$

The results in the last column of table 1 show that the values of $D_{\text {wcA }}$ reported by Kushik and Berne [10] can be represented by

$$
D_{\mathrm{WCA}}(\rho, T)=(0.9 \pm 0.05) D_{\mathrm{HS}}\left(\rho, T, \sigma_{\mathrm{B}}\right)
$$

To satisfy equation (1) exactly, the values of $\sigma_{\mathbf{B}}$ from equation (7) would need to be revised upwards by about 1 per cent.

The third column of table 1 tests the appealing notion that the effective hard sphere diameter $\sigma_{\mathrm{wCA}}$, which Weeks, Chandler and Andersen [4] have shown to model the structure and thermodynamics of the Lennard-Jones model, should also be the appropriate diameter for modelling diffusion.

$\sigma_{\mathrm{wCA}}$ is defined by equation (16) of [4]. The calculation requires values of the hard-sphere 'cavity-cavity' correlation function $y(r)$ in the range $0.7 \sigma<r \leqslant 2^{1 / 6} \sigma$, for which we used Ballance and Speedy's simple formula [22], along with Kolofa's 
Table 1. The self diffusion coefficient for a fluid defined by the Weeks, Chandler, Andersen (WCA) repulsive potential (equation 6) compared with $D_{\mathrm{HS}}(\rho, T, \sigma)$. In the third column the effective hard sphere diameter used, $\sigma_{\mathrm{wCA}}$, was calculated according to WCA criterion [4], as described in the text. In the fourth column $\sigma_{\mathrm{B}}$ is calculated from equation (7). Data from Kushik and Berne [10].

\begin{tabular}{cccc}
\hline$\rho \sigma_{\mathrm{LJ}}^{\mathbf{3}}$ & $k_{\mathrm{B}} T / \varepsilon$ & $D_{\mathrm{WCA}} / D_{\mathrm{HS}}\left(\sigma_{\mathrm{WCA}}\right)$ & $D_{\mathrm{WCA}} / D_{\mathrm{HS}}\left(\sigma_{\mathrm{B}}\right)$ \\
\hline 0.85 & 0.76 & 1.72 & 0.89 \\
0.85 & 4.64 & 1.52 & 0.94 \\
0.81 & 0.74 & 1.47 & 0.85 \\
0.75 & 0.91 & 1.37 & 0.90 \\
0.75 & 5.10 & 1.34 & 0.91 \\
0.65 & 1.42 & 1.24 & 0.92 \\
0.65 & 5.09 & 1.25 & 0.92 \\
0.30 & 1.58 & 1.05 & 0.90 \\
\hline
\end{tabular}

hard-sphere equation of state [23] to determine the pressure and chemical potential. The $y(r)$ function that results is probably the most accurate representation available over the required range of $r$. Table 1 shows that the values of $D_{\mathrm{Hs}}\left(\rho, T, \sigma_{\mathrm{wCA}}\right)$ which result are too small. In other words $\sigma_{\mathrm{wCA}}$ is too large to satisfy equation (1). The discrepancy is worst at the highest densities where the WCA theory is most accurate. The $\sigma_{\mathrm{WCA}}$ values are about 5 per cent bigger than $\sigma_{\mathrm{B}}$.

\section{Diffusion in methane}

Methane is the simplest real fluid for which precise and extensive diffusion measurements have been reported over a wide range of temperature and pressure [9]. To predict its diffusion coefficient we use literature values [24] of the LennardJones parameters $\sigma_{\mathrm{LJ}}=3.81 \AA$ and $\varepsilon / k_{\mathrm{B}}=148 \mathrm{~K}$ in the $\mathrm{LJ}$ potential function

$$
u(r)=4 \varepsilon\left(\left(\sigma_{\mathrm{LJ}} / r\right)^{12}-\left(\sigma_{\mathrm{LJ}} / r\right)^{6}\right)
$$

Boltzmann's diameter, from equation (5), is then

$$
\sigma_{\mathrm{B}}=\sigma_{\mathrm{LJ}}\left(2 /\left[1+\sqrt{ }\left(1+2 k_{\mathrm{B}} T / \varepsilon\right)\right]\right)^{1 / 6}
$$

Values of $D_{\mathrm{HS}}\left(\rho, T, \sigma_{\mathrm{B}}\right)$ calculated from (2) then agree with the measured values for methane to within 20 per cent.

The above Lennard-Jones parameters derive [24] from equation of state and viscosity measurements on the gas and it is unlikely that a non spherical molecule like methane can be represented accurately by the same parameters over the whole density range. An optimized choice of $\sigma_{\mathrm{LJ}}=4 \cdot 14 \AA$ and $\varepsilon / k_{\mathrm{B}}=30 \mathrm{~K}$ fits the diffusion data for liquid methane $\left(0.3<\rho \sigma^{3}<0.86\right)$ with a standard deviation of 2.3 per cent, which is better than the accuracy of the data (the two $D(p, T)$ data sets mismatch by 4 per cent where they meet [9]).

The difference between the literature values, of $\sigma_{\mathrm{LJ}}$ and $\varepsilon$, and the optimized values is not as significant as it looks. The values of $\sigma_{\mathrm{B}}$ that they yield agree to within 1.3 per cent over the temperature range of the diffusion measurements, as shown in figure 1 . With the optimized parameters (10) accurately represents the 


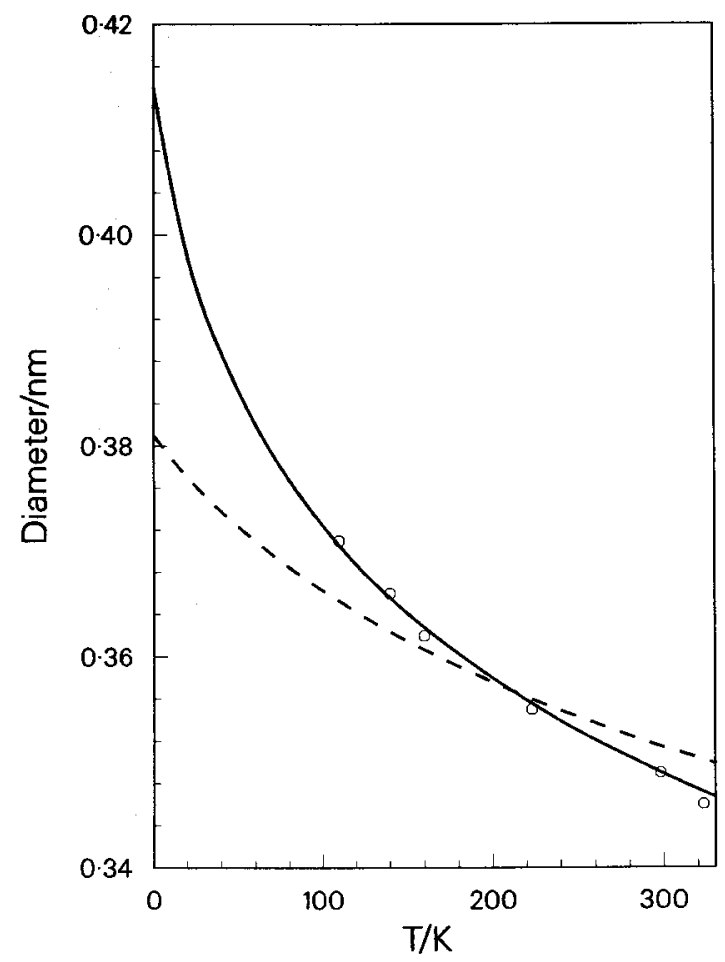

Figure 1. The effective hard sphere diameters $\sigma(T)$ for methane plotted against temperature. The points show best fit values obtained by fitting each isothermal data set [9] to equation (2). The dashed line shows values predicted by equation (10) using literature values [24] of $\sigma_{\mathrm{LJ}}$ and $\varepsilon / k_{\mathrm{B}}$. The solid line shows the values obtained from equation (10) when $\sigma_{\mathrm{LJ}}$ and $\varepsilon / k_{\mathrm{B}}$ are chosen so as to minimize the root mean square percentage difference between the combined data of [9] and equation (2).

values of $\sigma$ which give the best fit of equation (2) to the individual isothermal data sets.

Our fit of the liquid methane data to equation (2) using the optimized parameters also gives a good account of lower density measurements, as shown in figure 2. The fact that the experimental results form a single curve in figure 2 means that the isochoric temperature dependence of $D$ is accounted for the $\sqrt{ } T$ term in equation (2) and the temperature dependence of $\sigma_{\mathrm{B}}$ from equation (10). The other halomethanes studied show a stronger temperature dependence, which cannot be accounted for in that way. That led us to the following examination of the influence of the cohesive energy on diffusion.

\section{Influence of the cohesive energy on diffusion}

In this section we compare computer simulation results for diffusion in the square-well [11] and Lennard-Jones [10,12] model fluids with results for the hardsphere [19] and WCA core potentials [10] to demonstrate that the effect of the cohesive energy is to reduce diffusion, and to quantify the effect.

Note that we distinguish between the cohesive force and the cohesive energy. In the Weeks, Chandler, Andersen perturbation theory [4] the total potential function 


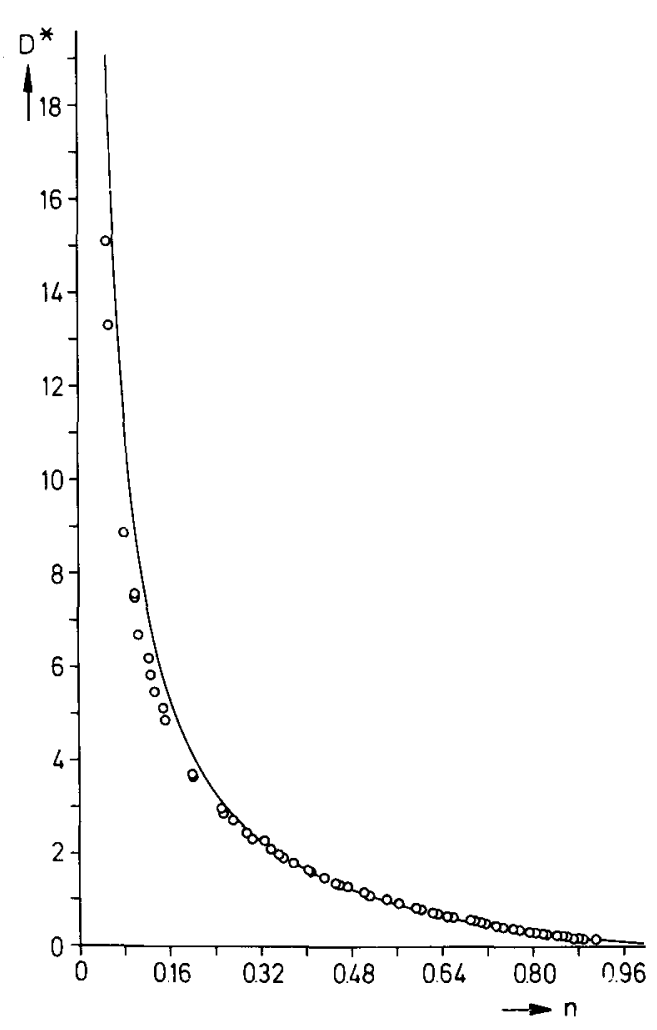

Figure 2. The reduced self diffusion coefficient of methane $D^{*}\left(D^{*}=D / D_{0}\right.$ equation (2)) plotted against the effective hard sphere density $\rho \sigma_{\mathbf{B}}^{3}$. The solid line represents equation (2) with $\sigma_{\mathrm{B}}$ given by equation (10) and using the parameter values $\sigma_{\mathrm{LJ}}=4 \cdot 14 \AA$ and $\varepsilon / k_{\mathrm{B}}=30 \mathrm{~K}$ which give the best fit to the liquid $\left(0.3<\rho \sigma_{\mathrm{B}}^{3}<0.86\right)$ and is extrapolated to lower densities.

is divided up into its attractive and repulsive parts according to the sign of the force $f(r)=-d u(r) / d r$. For the purpose of interpreting diffusion we choose to separate $u(r)$ into its positive and negative parts, that is according to the sign of $u(r)$ rather than the sign of $f(r)$. The cohesive energy, in the van der Waals or Boltzmann sense, is the energy difference between a system of molecules which interact with the full potential $u(r)$ and a system of molecules, at the same density, which interact with only the positive part of $u(r)$.

Figure 3 shows Alley and Alder's [9] results for diffusion in the square well (SW) model

$$
u(r)=\left\{\begin{aligned}
\infty, & r<\sigma \\
-\varepsilon, & \sigma<r<\lambda \sigma \\
0, & r>\lambda \sigma .
\end{aligned}\right.
$$

In this case there is no ambiguity in the choice of the diameter, $\sigma_{\mathbf{B}}=\sigma$. The results can be summarized empirically by

$$
\begin{aligned}
D_{\mathrm{SW}} & \simeq D_{\mathrm{HS}}\left(1-0.6 \varepsilon / k_{\mathrm{B}} T\right) \\
& \simeq D_{\mathrm{HS}} \exp \left(-\varepsilon / k_{\mathrm{B}} T\right) .
\end{aligned}
$$




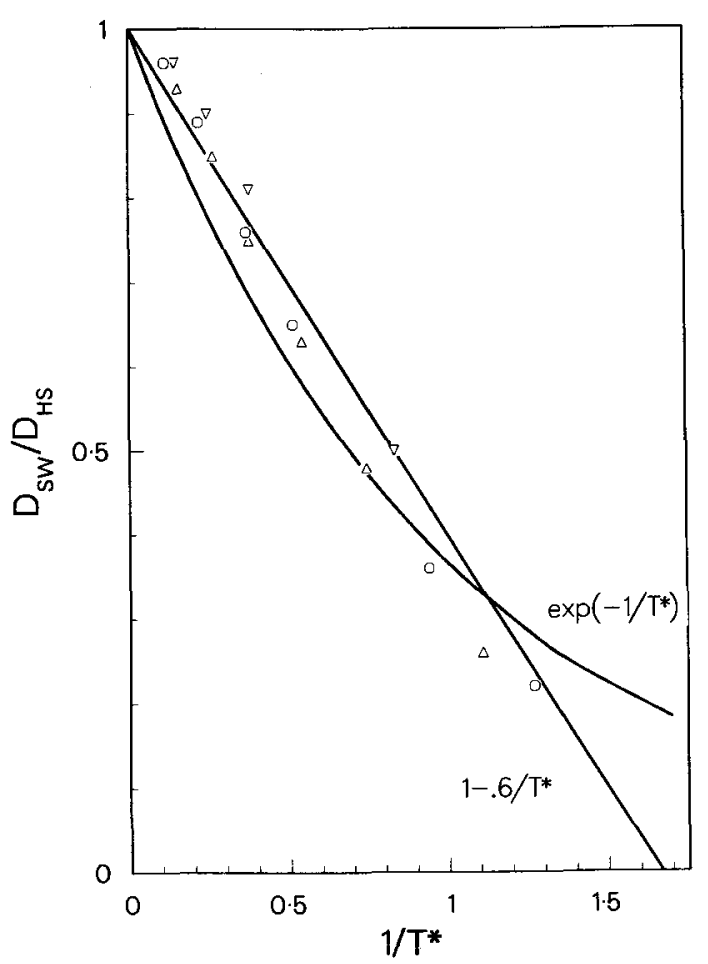

Figure 3. The self diffusion coefficient of a square well (equation (11)) fluid relative to that in hard spheres, plotted against $1 / T^{*} \equiv \varepsilon / k_{\mathrm{B}} T$. The lines represent equation (12). Data from Alley and Alder [11].

It can be argued [25] that because the SW potential is impulsive and does not conform to Boltzmann's description of the slowly varying cohesive force quoted at the beginning of this paper, the reduction in $D_{\mathrm{sw}}$ due to the well is not a general feature of realistic attractive potentials. Against that argument, Alley and Alder [9] show that the reduction can be predicted by a 'local density' assumption as follows: if it is assumed that $D_{\mathrm{sw}}(\rho)$ is the same as $D_{\mathrm{Hs}}\left(\rho_{1}\right)$, where $\rho_{1}$ is the local density inside the square well, then the slope of about 0.6 of the straight line drawn in figure 3 is predicted (in the high temperature limit). Qualitatively, that argument applies equally well to, say, a Lennard-Jones system although quantitatively there is, with a continuous potential, some arbitrariness in the choice of the region over which the local density is defined.

While the square well system shows the most dramatic influence of the negative part of $u(r)$ on $D$ we need not rely on it for evidence that the effect is a general feature of realistic potentials.

Levesque and Verlet [12] studied diffusion in the Lennard-Jones system in 1970. Their conclusion was that $D_{\mathrm{LJ}} \simeq D_{\mathrm{HS}}$ if the hard sphere diameter was chosen by the criterion that the height of the first peak in the structure factors for the two systems should match. Weeks, Chandler and Andersen [4] showed that criterion to be accurately satisfied by their prescription for choosing the diameter $\sigma_{\mathrm{wCA}}$ equation (16) of [4].

Figure 4 shows, as open symbols the ratio $D_{\mathrm{LJ}} / D_{\mathrm{HS}}\left(\sigma_{\mathrm{wCA}}\right) \cdot \sigma_{\mathrm{wCA}}$ was recalculated as described in $\S 3$. Again, it is clear that $\sigma_{\mathrm{wCA}}$ is too large to satisfy equation (1). 


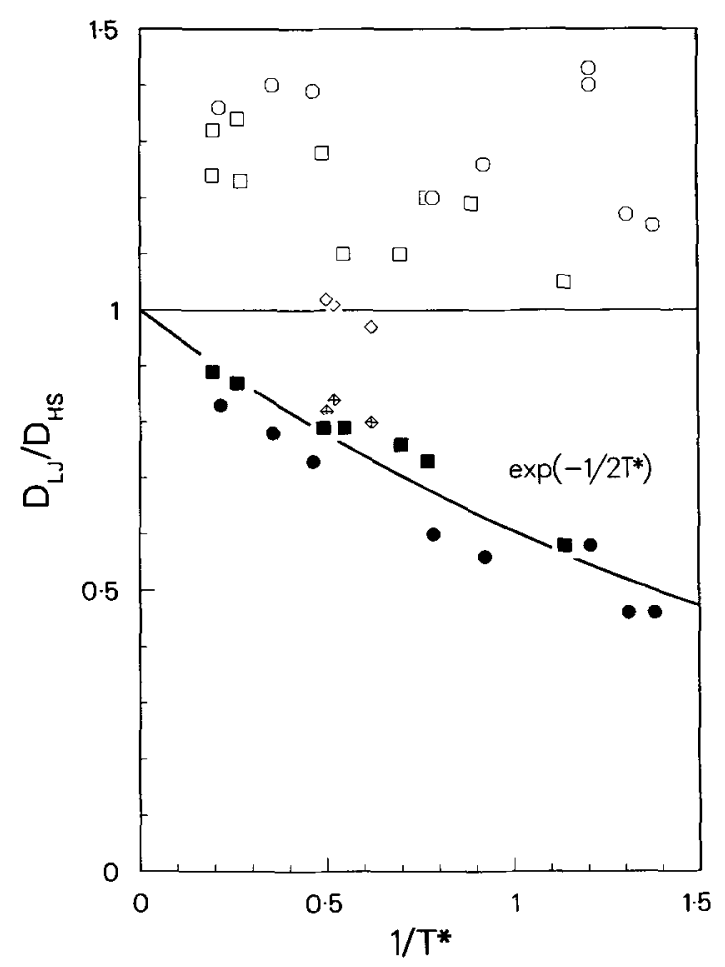

Figure 4. The self diffusion coefficient for the Lennard-Jones fluid (equation (9)) relative to that for hard spheres, plotted against $1 / T^{*} \equiv \varepsilon / k_{\mathrm{B}} T$. Open symbols show results when the WCA prescription [4] is used to estimate the effective hard sphere diameter $\sigma_{\mathrm{wCA}}$. Filled symbols show results when Boltzmann's diameter, $\sigma_{\mathbf{B}}$, from equation (10) is used. The solid line represents equation (13). Symbols distinguish different densities: $\rho \sigma_{\mathrm{LJ}}^{3}=0.85$ and 0.844 , circles; $\rho \sigma_{\mathrm{LJ}}^{3}=0.75$ and 0.65 , squares; $\rho \sigma_{\mathrm{LJ}}^{3}=0.3$ and 0.4 , diamonds. Data from Levesque and Verlet [12].

The filled symbols show the ratio $D_{\mathrm{LJ}} / D_{\mathrm{HS}}\left(\sigma_{\mathrm{B}}\right)$ with $\sigma_{\mathrm{B}}$ calculated from equation (10). In this case there is a systematic trend which can be represented by

$$
\begin{aligned}
D_{\mathrm{LJ}} & \simeq D_{\mathrm{HS}}\left(\sigma_{\mathrm{B}}\right)\left(1-0 \cdot 4 \varepsilon / k_{\mathrm{B}} T\right) \\
& \simeq D_{\mathrm{HS}}\left(\sigma_{\mathrm{B}}\right) \exp \left(-\varepsilon / 2 k_{\mathrm{B}} T\right) .
\end{aligned}
$$

The exponential form represents Levesque and Verlet's [12] values of $D_{L J}$ with a standard deviation of 6.8 per cent and Kushik and Berne's [10] values of $D_{\mathrm{LJ}}$ with a standard deviation of 6.4 per cent. Heyes [26] presents more extensive $D_{\mathrm{LJ}}$ data but they seem to be much less precise. For example, over the density and temperature range for which the accuracy of equation (13) is established, by the data of Levesque and Verlet [12] and Kushik and Berne [10], Heyes [26] results show a standard deviation of 13 per cent from equation (13), increasing to 16 per cent when Heyes higher density results are included.

Note that the exponential term in equation (13) accommodates not only the effect of the potential well but also the effect of any error in the choice of $\sigma_{\mathrm{B}}$. Those two effects are not separable without some further assumption. One can, for example, compare $D_{\mathrm{LJ}}$ with $D_{\mathrm{wCA}}$ and attribute the difference to attractive forces, or one could compare $D_{\mathrm{LJ}}$ with $D_{\mathrm{SS}}$, the diffusion coefficient for the soft sphere poten- 
tial $u(r)=4 \varepsilon\left(\sigma_{\mathrm{L} J} / r\right)^{12}$. Those comparisons are independent of $\sigma_{\mathrm{B}}$ but they yield different measures of the effect of attractive forces.

The value of $\sigma_{\mathrm{B}}$ from equation (10) is the solution of equations (5) and (9) for the full Lennard-Jones potential. However, it is also the solution of equations (5) and (14) for the positive part of the $\mathrm{LJ}$ potential

$$
u(r)= \begin{cases}4 \varepsilon\left(\left(\sigma_{\mathrm{LJ}} / r\right)^{12}-\left(\sigma_{\mathrm{LJ}} / r\right)^{6}\right), & r<\sigma_{\mathrm{LJ}}, \\ 0, & r>\sigma_{\mathrm{LJ}}\end{cases}
$$

The term $D_{\mathrm{HS}}\left(\sigma_{\mathrm{B}}\right)$ in (13) is therefore a prediction of the diffusion coefficient of a system of molecules interacting with the potential defined by equation (14). No data are available to verify the accuracy of that prediction directly, but since the positive LJ potential (equation (14)) is steeper and narrower than the repulsive WCA potential (equation (7)) it is likely that our prediction of its diffusion coefficient is at least as accurate as our prediction of $D_{\mathrm{wCA}}$, shown in the last column of table 1 .

With that identification of $D_{\mathrm{HS}}\left(\sigma_{\mathrm{B}}\right)$, the exponential term in equation (13) then represents the effect on $D_{\mathrm{LJ}}$ of turning on the negative energy well of the complete Lennard-Jones potential. That assignment of the exponential term is supported by the similarity of its form (figure 4) to the square well case (figure 3) where its assignment is not dependent on any assumption.

While our object is to relate diffusion in real liquids to $D_{\mathrm{HS}}$, it may also be useful, for Lennard-Jones systems, to relate $D_{\mathrm{LJ}}$ to $D_{\mathrm{WCA}}$, the diffusion coefficient in the WCA core system (equation (6)). Kushik and Berne [10] have calculated both $D_{\mathrm{LJ}}$ and $D_{\mathrm{WCA}}$ at the densities and temperatures listed in table 1 . Their results can be represented by

$$
D_{\mathrm{LJ}}=D_{\mathrm{WCA}}\left(1-0 \cdot 14 \varepsilon / k_{\mathrm{B}} T\right),
$$

with a standard deviation of 3.8 per cent. This result shows that when the reference system is chosen by the repulsive force criterion $f(r)>0$, rather that by the positive energy criterion $u(r)>0$, turning on the remainder of the $L J$ potential has a smaller effect on $D_{\mathrm{LJ}}$, but not a negligible effect.

\section{Diffusion in the halomethanes}

Previous analyses of diffusion in the halomethanes [13-17] and other simple liquids [18] have established that, in contrast to methane, they do not conform to (1). The usual explanation is that provided by Chandler's 'rough hard sphere' model, which attributes a reduction in diffusion to the dissipation of velocity correlations by inelastic effects like translation-rotation coupling. Chandler [7] proposes that

$$
D=A D_{\mathrm{HS}}(\sigma),
$$

where $A \leqslant 1$ is a temperature independent translation-rotation coupling constant. When experimental data are fitted to equation (16) to obtain best fit values of $A$ and $\sigma$ for each isothermal data set, the values of $A$ which result show a strong temperature dependence [13-18]. Hence the temperature dependence is stronger than can be accounted for by the $\sqrt{ } T$ dependence of $D_{\mathrm{Hs}}$ and the temperature dependence of $\sigma$.

Figure 4 shows that an Arrhenius type temperature dependence is manifested by the Lennard-Jones system and is therefore to be expected in real liquids. It accounts 
Table 2. Diffusion data fitted to $D=D_{\mathrm{HS}}(T, \rho, \sigma) e-\varepsilon / 2 k_{\mathrm{B}} T$ with $\sigma=\sigma_{\mathrm{LJ}}(2 /(1+\sqrt{ }(1$ $\left.\left.\left.+2 k_{\mathrm{B}} T / \varepsilon\right)\right)\right)^{1 / 6}$ and $D_{\mathrm{HS}}$ given by equation (2).

\begin{tabular}{|c|c|c|c|c|c|c|}
\hline Substance & Source & $T / \mathbf{K}$ & $\rho \sigma^{3}$ & $\sigma_{\mathbf{L}} / \mathrm{nm}$ & $\left(\varepsilon / k_{\mathbf{B}}\right) / \mathbf{K}$ & $\%$ Std. dev. \\
\hline $\mathrm{CH}_{4}$ & 9 & $110-328$ & $0.33-0.89$ & $\begin{array}{l}0.414 \dagger \\
0.412\end{array}$ & $\begin{array}{l}30 \dagger \\
29\end{array}$ & $\begin{array}{l}2 \cdot 3 \dagger \\
4.0\end{array}$ \\
\hline $\mathrm{CH}_{3} \mathrm{Cl}$ & $13 \ddagger$ & $210-440$ & $0.53-0.88$ & 0.436 & 129 & $8 \cdot 2$ \\
\hline $\mathrm{CH}_{2} \mathrm{Cl}_{2}$ & $13 \ddagger$ & $186-406$ & $0.65-1.02$ & 0.479 & 162 & $3 \cdot 3$ \\
\hline $\mathrm{CHCl}_{3}$ & $13 \ddagger$ & $233-397$ & $0.73-0.98$ & 0.534 & 107 & $4 \cdot 5$ \\
\hline $\mathrm{CCl}_{4}$ & 14 & $283-328$ & $0.87-0.99$ & 0.575 & 145 & $6 \cdot 3$ \\
\hline $\mathrm{CH}_{3} \mathrm{~F}$ & $15_{+}^{+}$ & $153-440$ & $0.21-0.90$ & 0.405 & 56 & $8 \cdot 5$ \\
\hline $\mathrm{CHF}_{3}$ & $16 \ddagger$ & $142-433$ & $0.31-0.99$ & 0.434 & 86 & $6 \cdot 6$ \\
\hline $\mathrm{CHClF}_{2}$ & $17 \ddagger$ & $147-383$ & $0.49-1.04$ & 0.461 & 164 & $6 \cdot 8$ \\
\hline
\end{tabular}

$\dagger$ Using the equation $D=D_{\mathrm{HS}}(T, \rho, \sigma)$ for methane $(\S 3)$.

\$ Some densities were extrapolated graphically from measured values as discussed in the references.

quantitatively for the reduction in $D$ which has hitherto been attributed to inelastic effects.

Table 2 summarizes the results of fitting measured diffusion coefficients for the halomethanes with equation (13), using (10) for $\sigma_{\mathrm{B}}$. The quality of the fits is illustrated in figures 5-8.

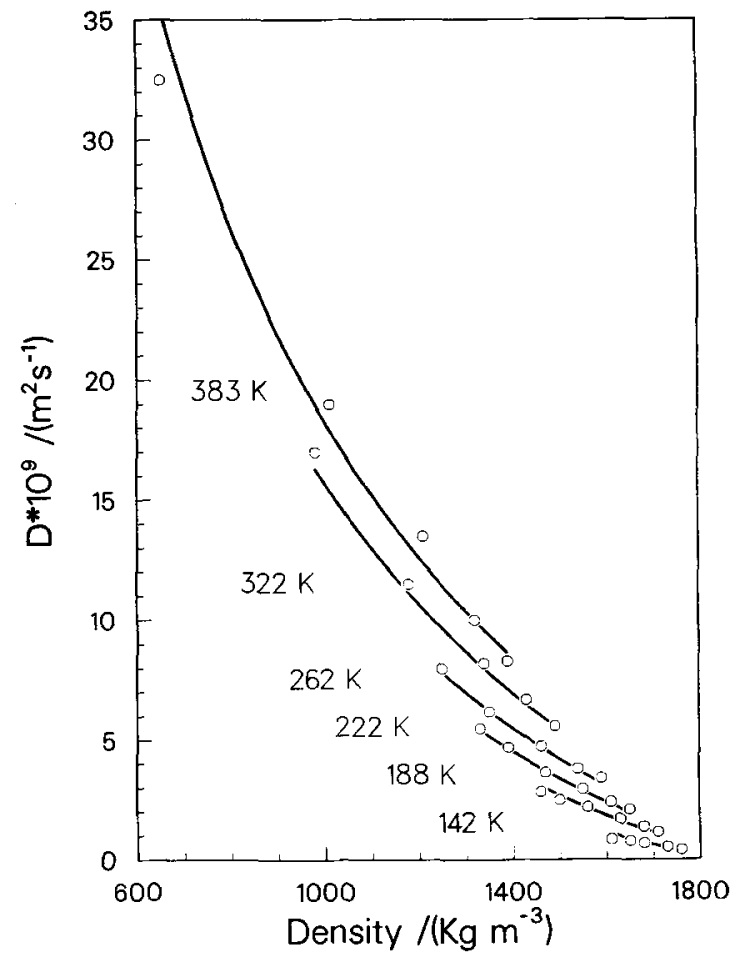

Figure 5. The self diffusion coefficient of $\mathrm{CF}_{3} \mathrm{H}$ plotted against density. For clarity several isothermal data sets are omitted. Densities below $273 \mathrm{~K}$ are extrapolated. The solid lines represent equation (13) with $\sigma_{\mathrm{B}}$ given by equation (10) using the parameter values listed in table 1 . 


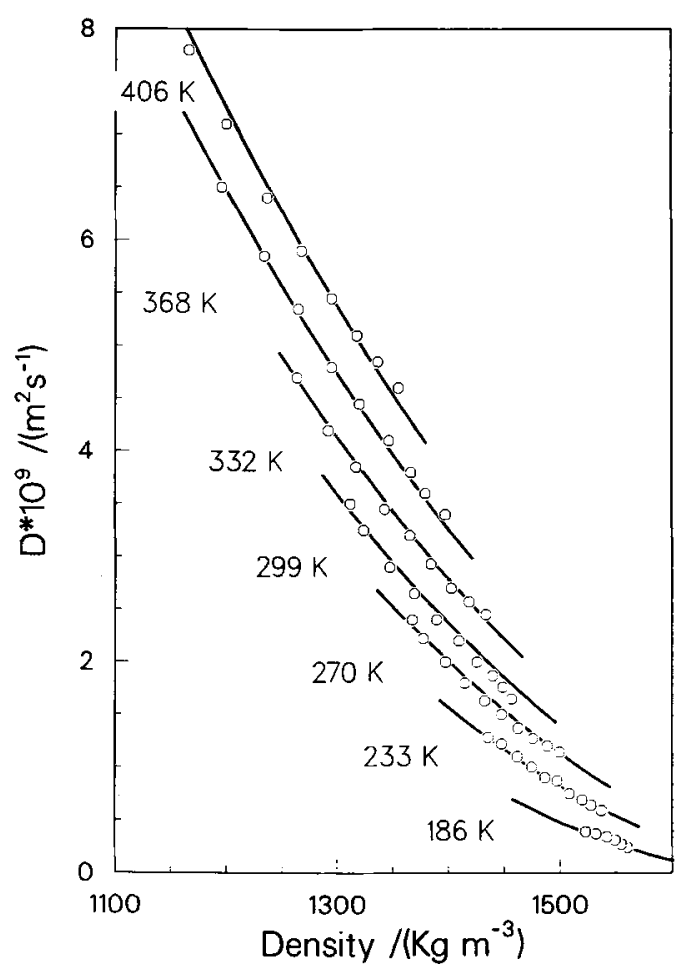

Figure 6. The self diffusion coefficient of $\mathrm{CH}_{2} \mathrm{Cl}_{2}$ plotted against density as in figure 5 . Densities below $300 \mathrm{~K}$ are extrapolated.

The standard deviations listed in table 1 are comparable to the estimated precision of the measurements ( 5 to 10 per cent), but there are some systematic deviations of up to 20 per cent. In most cases density measurements are not available over the whole temperature and pressure range of the diffusion measurements and graphical extrapolations from available density data have been used [13-17]. In the worst cases the extrapolated densities are uncertain to 1 or 2 per cent corresponding to uncertainties of 10 to 20 per cent in $D$ at high density, and the worst systematic deviations occur in those cases.

The intermolecular potentials of the substances listed in table 2 can be, at best, crudely approximated by the Lennard-Jones function (equation (9)) and that approximation is the most likely source of the systematic deviations. In particular, the (orientationally averaged) repulsive potential between the globular polyatomic molecules is probably steeper, on the molecular length scale, than that between atoms. Thus the $\mathrm{LJ}$ potential tends to overestimate the temperature dependence of $\sigma_{\mathrm{B}}$ (equation (10)) and better fits (standard deviations listed in table 2 are reduced by 2 percentage points in some cases) are obtained by replacing the parameter $\varepsilon$ in equation (10). by $2 \varepsilon$, which, effectively, gives a steeper repulsive potential with the same well depth.

The density of the measurements is higher than the highest density $\rho \sigma_{\mathrm{Lj}}^{3}=0.85$ to which the accuracy of equation (13) is established by the precise simulation data of Levesque and Verlet [12] and Kushik and Berne [10]. Heyes [26] computed values of $D_{\mathrm{LJ}}$ at higher densities are systematically lower than the predicted values but they are not sufficiently precise to determine a quantitative trend. 


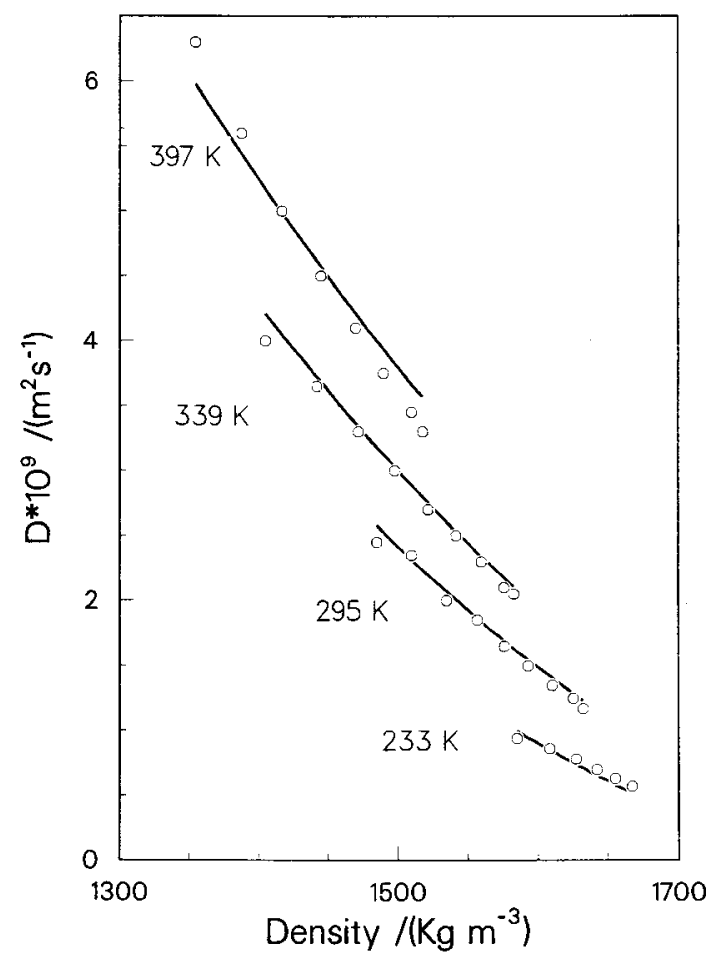

Figure 7. The self diffusion coefficient of $\mathrm{CHCl}_{3}$ plotted against density as in figure 5 . Densities below $300 \mathrm{~K}$ are extrapolated.

The experimental data were also fitted to equation (15) using equation (8) for $D_{\text {wCA }}$. The standard deviations were significantly higher than those in table 2 except for methane (std dev $=2.5$ per cent). The worse fit in this case is attributable to the inadequacy of the LJ potential. The diameter $\sigma_{\mathbf{B}}$ for the WCA core potential (equation (7)) is more sensitive to the form of the potential than is the diameter $\sigma_{\mathrm{B}}$ for the positive part of the LJ potential (equation (10)) and it has a stronger temperature dependence.

We conclude that equation (13) offers a plausible, physically sound and reasonably precise two parameter description of the magnitude, temperature and density dependence of diffusion in simple liquids. Some quantitative improvement is no doubt possible, for data fitting purposes, at the expense of extra parameters, but there is no indication that the asymmetric polyatomic molecules studied show any qualitative, or significant quantitative, difference from the Lennard-Jones model system. In particular, the observation that diffusion in these fluids is less than that in the corresponding hard sphere system is explained by the influence of the negative energy potential well. That reduction is present in the Lennard-Jones system and it is not necessary to invoke inelastic effects like translation-rotation coupling to explain it.

\section{Summary}

Our development of Boltzmann's observation: that the collisional trajectories of repelling molecules are not essentially different from the trajectories of a pair of hard spheres with diameters equal to the distance of closest approach; leads directly 


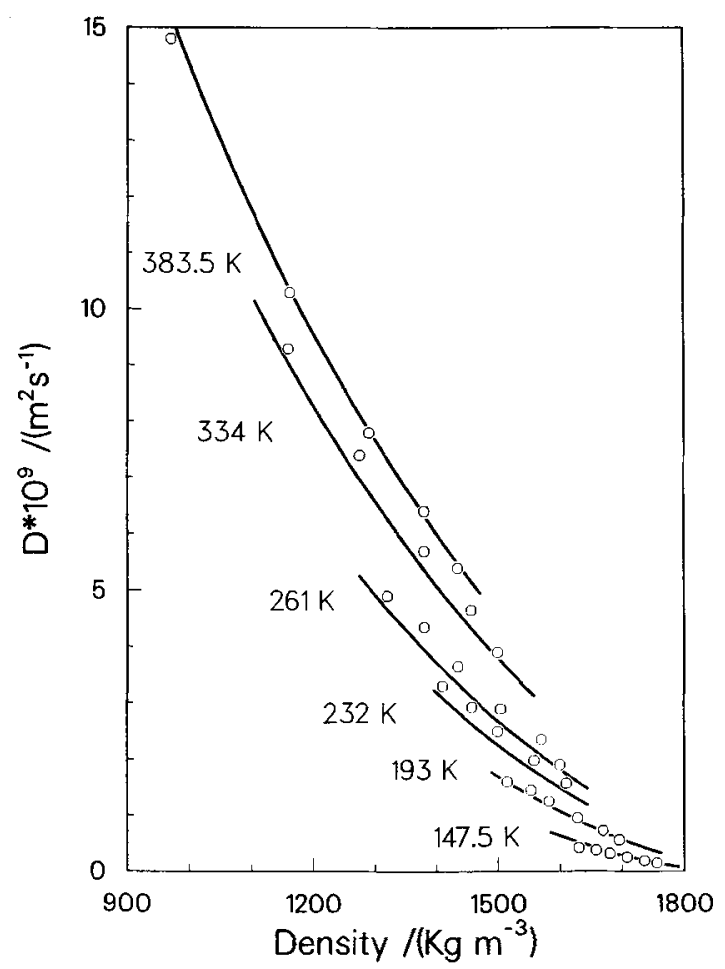

Figure 8. The self diffusion coefficient of $\mathrm{CF}_{2} \mathrm{HCl}$ plotted against density as in figure 5 . Densities below $250 \mathrm{~K}$ are extrapolated.

to quantitatively accurate predictions of diffusion in methane and in the Weeks, Chandler-Andersen repulsive reference system.

But his other suggestion: that molecular dynamics are not affected by the van der Waals cohesion energy; is only approximate. The results of computer simulation studies allow us to quantify the effect for the square well and Lennard-Jones fluids and, thereby, to represent $D_{\mathrm{L}}$ by equation (13). The calculated diffusion coefficients of several model systems are simply interrelated when Boltzmann's diameter is used to take account of differences in the positive parts of their intermolecular potentials.

Measured self diffusion coefficients of the halomethanes have essentially the same magnitude and temperature and density dependence as $D_{\mathrm{LJ}}$ and do not reveal the influence of any inelastic effects associated with molecular anisotropy. To reveal such effects will require more precise and extensive measurements, or measurements on more asymmetric systems.

Financial support by the Deutsche Forschungsgemeinschaft and the Fonds der Chemischen Industrie is gratefully acknowledged. F.X.P. thanks the FriederichEbert-Stiftung for a scholarship. We thank David Chandler for helpful advice

\section{References}

[1] BoltzmanN, L., 1964, Lectures on Gas Theory, translated by S. Brush (University of California Press) p. 220. Boltzmann adds (p. 375) that van der Waals did not make such an assumption explicitly and believed such a force law to be improbable. 
[2] Zwanzig, R., 1954, J. chem. Phys., 22, 1420. Longuet-Higgins, H. C., and Widom, B., 1970, J. chem. Phys., 52, 2315.

[3] Barker, J. A., and Henderson, D., 1967, J. chem. Phys., 47, 4714. Henderson, D., and BARKer, J. A., 1970, J. chem. Phys., 52, 2315.

[4] Weeks, J. D., Chandler, D., and Andersen, H. C., 1971, J. chem. Phys., 54, 5237. Chandler, D., Weeks, J. D., and Andersen, H. C., 1983, Science, N.Y., $220,787$.

[5] Dymond, J. H., 1974, Physica, 75, 100; 1985, Chem. Soc. Rev., 14, 317.

[6] Tyrell, H. J. V., and Harris, K. R., 1984, Diffusion in Liquids (Butterworths).

[7] Chandler, D., 1974, J. chem. Phys., 60, 3500; 1975, Ibid., 62, 1358.

[8] BoltzmanN, L., 1964, Lectures on Gas Theory, translated by S. Brush (University of California Press), p. 169.

[9] Harris, K. R., and Trappeniers, N. J., 1980, Physica A, 104, 262.

[10] KushiK, J., and Berne, B. J., 1973, J. chem. Phys., 59, 3732.

[11] Alley, W. E., and Alder, B. J., 1975, J. chem. Phys., 63, 3764.

[12] LeVesque, D., and Verlet, L., 1970, Phys. Rev. A, 2, 2514.

[13] Prielmeier, F. X., and Lüdemann, H.-D., 1986, Molec. Phys., 58, 593.

[14] McCool, M. A., and Woolf, L. A., 1972, Chem. Soc. Faraday Trans. I, 68, 1971.

[15] Lang, E. W., Prielmeier, F. X., Radkowitsch, H., and Lüdemann, H. D., 1987, Ber. Bunsenges. phys. Chem., 91, 1017.

[16] Prielmeier, F. X., Lang, E. W., and Lüdemann, H.-D., 1984, Molec. Phys., 52, 1105.

[17] VARDAG, T., 1987, Diplomarbeit, Regensburg.

[18] Easteal, A. J., and Woolf, L. A., 1984, Physica B, 124, 173, 182.

[19] SPEedY, R. J., 1987, Molec. Phys., 62, 509.

[20] SPEEDY, R. J., 1986, Ann. N.Y. Acad. Sci., 484, 214.

[21] Chapman, S., and Cowling, T. G., 1960, The Mathematical Theory of Non Uniform Gases (Cambridge University Press), equation (5.3.5).

[22] BallanCe, J. A., and SpeEdy, R. J., 1985, Molec. Phys., 54, 1035.

[23] Boublik, T., and Nezbeda, I., 1986, Colln. Czech. chem. Commun., 51, 2301 (equation 4.46).

[24] Hirschfelder, J. O., CurTis, C. F., and BiRd, R. B., 1954, Molecular Theory of Gases and Liquids (Wiley).

[25] ChANDLER, D. (personal communication).

[26] HeYES, D. M., 1983, J. chem. Soc. Faraday II, p. 1741. 